\title{
Special section on nonlinear dynamical aspects of edge computing and neuromorphic hardware
}

Nonlinear dynamics provides us with an essential viewpoint for further development of edge computing and neuromorphic hardware towards new paradigms of computing. With this shared motivation, six papers contributed to this Special Section address various topics of computing, ranging from neural network models to hardware implementation. We hope that this section contributes to further progress in related research fields.

This Special Section was proposed as a part of joint activities of two research projects entitled "Computing technology based on spatiotemporal dynamics of photonic neural networks" and "Fundamentals of spintronics-based edge computing hardware" in CREST Research Area "Technology for Computing Revolution for Society 5.0" of Japan Science and Technology Agency (JST).

The editors for the Special Section would like to express their sincere thanks to all of the authors for their contributions. They also thank all of the reviewers, guest editorial members, and editorial staff of the NOLTA journal for their supports on publishing this Special Section.

\section{Hideyuki Suzuki, Shigeo Sato}

Guest Editors for the Special Section

\author{
Editorial Members for the Special Section \\ Guest Co-Editors: \\ Hideyuki Suzuki (Osaka Univeristy) \\ Shigeo Sato (Tohoku University) \\ Guest Associate Editors: \\ Kantaro Fujiwara (The University of Tokyo) \\ Yuichi Katori (Future University Hakodate) \\ Timothée Leleu (The University of Tokyo) \\ Jordi Madrenas (Technical University of Catalunya) \\ Naoya Onizawa (Tohoku University) \\ Hakaru Tamukoh (Kyushu Institute of Technology) \\ Takaharu Yaguchi (Kobe University) \\ Hideaki Yamamoto (Tohoku University) \\ Secretaries of the Special Section: \\ Sho Shirasaka (Osaka University) \\ Satoshi Moriya (Tohoku University) \\ Advisory: \\ Masaharu Adachi (Tokyo Denki University)
}

